\title{
Laser ionization and radio frequency sustainment of high-pressure seeded plasmas
}

\author{
K. L. Kelly, J. E. Scharer, ${ }^{\text {a) }}$ E. S. Paller, and G. Ding \\ Department of Electrical and Computer Engineering, University of Wisconsin-Madison, Madison, \\ Wisconsin 53706
}

(Received 12 December 2001; accepted for publication 1 May 2002)

\begin{abstract}
The feasibility of using a photoionized, low-ionization potential organic seed gas to initiate a high pressure plasma discharge is examined and compared to radio frequency breakdown of high pressure argon alone. The seed gas, tetrakis(dimethylamino)ethylene, which has an ionization potential of $6.1 \mathrm{eV}$ is ionized by an ultraviolet laser through $6.4 \mathrm{eV}$ photon absorption, and forms a plasma column inside a vacuum chamber. The plasma absorbs additional power through inductive coupling of $13.56 \mathrm{MHz}$ helical antenna radio frequency wave fields to the plasma through electron acceleration, ionization, and collisional damping. Laser initiation of 2-6 mTorr of the seed gas in 1-150 Torr of argon is accomplished and produces steady-state line-average plasma densities of $n_{e} \approx 4 \times 10^{12} \mathrm{~cm}^{-3}$ in a volume of $300 \mathrm{~cm}^{3}$. The two-body recombination coefficient of the organic seed gas and its optimum partial pressure when mixed with argon are experimentally determined and analyzed. Particle loss and power requirements for maintaining the discharge are evaluated by examining ionization, diffusion, and recombination processes. (C) 2002 American Institute of Physics. [DOI: 10.1063/1.1487905]
\end{abstract}

\section{INTRODUCTION}

There has been considerable interest in producing highdensity, low-power plasma sources in gases that approach atmospheric pressure. The applications of these plasma sources call for densities of $n_{e} \sim 10^{11-13} \mathrm{~cm}^{-3}$ while minimizing power requirements. The pressures for these applications range from 100 up to and beyond 760 Torr and the minimum plasma volume is on the order of hundreds of cubic centimeters. Previous experiments pertaining to induction coils $^{1}$ required power densities of approximately $10-20 \mathrm{~W}$ per cubic centimeter to maintain the plasma at steady state at $n_{e}>10^{13} \mathrm{~cm}^{-3}$ as the pressure was continuously raised from 1 to 760 Torr of air. Near atmospheric pressure plasmas may have applications in reducing drag on supersonic vehicles, reducing radar cross sections by instantly forming a microwave absorbing or reflecting medium about an aircraft, ${ }^{2-4}$ in decontaminating environmental waste and gaseous pollution, specifically, hydrocarbons and E coli and bacillus, and in surface sterilization, ${ }^{5}$ as well as thin film and materials processing. ${ }^{6}$

One issue particular with regard to these high-density, high-pressure sources concerns the power required to create electrons in a density range where two-body and three-body recombinations are significant loss mechanisms. This can be influenced to some extent by appropriate choices of the working gas or by injecting another gas into air. Noble gases make exceptional choices since they are not reactive to other chemicals and typically have recombination coefficients which are lower than more complex molecules. They are also monoatomic, meaning that collisions between electrons and neutrals do not excite the internal rotational and vibration

${ }^{a)}$ Electronic mail: scharer@cptc.wisc.edu degrees of freedom associated with polyatomic molecules, thereby decreasing the energy cost per electron-ion pair created. In addition, they do not readily form negative ions, which increases the efficiency of plasma production and makes modeling of the plasma dynamics simpler.

Schuetze et al. ${ }^{7}$ has investigated a plasma torch for materials processing. They indicated that, although a $300 \mathrm{kV}$ potential was necessary to initiate an arc discharge in the atmosphere, only $100 \mathrm{~V}$ is needed to maintain the discharge. They referred to experiments by $\mathrm{Kim}$ and $\mathrm{Hong}^{8}$ that involved a torch with electron densities of $3 \times 10^{17} \mathrm{~cm}^{-3}$ and electron temperatures of $7-9 \mathrm{eV}$ in a variety of rare gas mixtures in volumes of 100-1000 cubic centimeters. Neutral gas temperatures of $3000{ }^{\circ} \mathrm{C}$ were measured with a rf input power of $200 \mathrm{~kW}$.

Eckert et al. ${ }^{1}$ used an atmospheric pressure plasma in both argon and air to study the emission spectrum given by a high pressure plasma. Following the work of Babat, ${ }^{9}$ they created a plasma using an inductive coil at a pressure of 1 Torr, and slowly increased the neutral pressure and rf power until they could open the plasma chamber to the atmosphere. To protect the quartz chamber from damage by heat and to help stabilize the discharge, they flowed the gas in a vortex, essentially forming a thermal barrier between the hot plasma and the chamber wall. The input power required to maintain the discharge was $15-50 \mathrm{~kW}$ at $4 \mathrm{MHz}$ to create and sustain a plasma with a volume of about 2500 cubic centimeters $\left(6-10 \mathrm{~W} / \mathrm{cm}^{3}\right)$.

Previous experiments by our group ${ }^{10-12}$ that pertained to an UV laser-produced plasma in tetrakis(dimethylamino)ethylene (TMAE) have shown that a plasma with a maximum electron density of $n_{e} \approx 4 \times 10^{13} \mathrm{~cm}^{-3}$ can be created in the organic seed gas, and that plasma lifetimes of TMAE have been extended by the addition of air components, specifically 
nitrogen (Ref. 12) and argon. The increase in the plasma lifetime has been attributed to the presence of superexcited states of the TMAE molecule which may readily make ionizing collisions with the nitrogen molecules at high pressure. Ten $\mathrm{GHz}$ microwave reflectivity experiments ${ }^{10}$ of a sheet plasma formed by the laser have been used to determine the TMAE plasma density and two-body recombination rates. Fast Langmuir probe measurements in low pressure TMAE (Ref. 11) have also been carried out to determine an initial electron temperature of approximately $0.3 \mathrm{eV}$ shortly after the laser pulse and the recombination rates. We have found that the liquid form of TMAE is compatible with air over long times and that the vapor form can be used in air for minutes. In some cases the emission from the gas phase reaction between TMAE and oxygen is very long lived and is measurable even after $30 \mathrm{~min} .{ }^{13}$ Thus TMAE can be considered to be a seed in air by pulsed injection and fast laser or ultraviolet (UV) flash tube ionization for plasma initiation.

In this article we examine the feasibility of ionizing an organic molecule which has a low ionization potential and is seeded in a high pressure gas. Ionizization of the seed gas is accomplished by $(193 \mathrm{~nm})$ ultraviolet laser photon absorption and sustainment of the high plasma density occurs by coupling $13.56 \mathrm{MHz}$ radio frequency power to the plasma.

To this end, radio frequency wave modes have been studied theoretically and experimentally to determine which antenna configuration is optimal for sustaining the highdensity laser-produced plasma. The theoretical study includes the use of the computer code ANTENAII ${ }^{14-16}$ which includes effects associated with one-dimensional (radial) density profiles for various antenna configurations, plasma densities, pressures, and magnetic field strengths. The coupling parameters of the antenna can be characterized by an overall impedance $Z_{\text {ant }}=R+i X$. From these parameters, a quality factor, $Q=X / R$ is determined and the optimum antenna will have a low overall $Q$ indicating that more energy is being transferred from the antenna to the plasma and less energy is being stored in the reactive fields.

The concept is to create a large volume ( $V$ $\sim 100-1000 \mathrm{~cm}^{3}$ ) plasma using a short pulse ( $\tau=20 \mathrm{~ns}$ ) ultraviolet laser to ionize the organic molecule tetrakis(dimethylamino)ethylene, or TMAE. The choice of TMAE as the seed gas is attractive for two particular reasons. First, the ionization potential for TMAE is $6.1 \mathrm{eV}$, much lower than the ionization potential of any of the noble gases which have ionization potentials in the range of $15 \mathrm{eV}$. At high pressures, the electron temperature decreases, and there is an absence of high energy particles in the tail of the electron energy distribution. Because of this, having a molecule with a low ionization potential is quite desirable. Second, the TMAE molecule has a large cross section for photoionization by ultraviolet light, meaning that the plasma can be initiated in large volumes by illuminating the plasma chamber.

Flash bulbs, spark gaps, and other sources of UV radiation could potentially be used to initiate the plasma. The availability of an argon-fluoride excimer laser which emits high power ( $300 \mathrm{~mJ} /$ pulse) $193 \mathrm{~nm}, E=h \nu=6.4 \mathrm{eV} /$ photon UV radiation with a relatively high power density (crosssectional area $A=4 \mathrm{~cm}^{2}$ ) makes TMAE an excellent choice

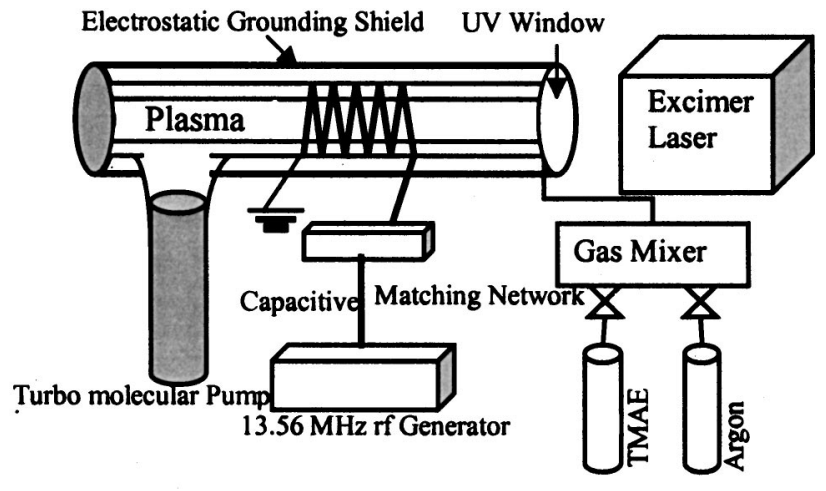

FIG. 1. Schematic of the rf-sustained laser-produced plasma experiment.

as the working seed gas. Once the plasma is initiated, it is possible to sustain the discharge by inductive radio frequency power at a much lower level than if the plasma were ionized by radio frequency power alone.

\section{LAB APPARATUS}

\section{A. Vacuum chamber and laser}

Figure 1 shows the apparatus used in the experiment. An excimer laser (Lumonics Pulsemaster PM-842) produces a $193 \mathrm{~nm}$ UV beam. The $\left(\frac{1}{2} \max \right)$ pulse width is $20( \pm 2) \mathrm{ns}$ with a maximum laser energy of $300 \mathrm{~mJ}$. The laser output cross section is $2.8 \mathrm{~cm} \times 1.4 \mathrm{~cm}$. Previous experiments using this laser ${ }^{10,11,17}$ have demonstrated that a high-density TMAE plasma with a high degree of uniformity $\left(\Delta n_{e} / n_{e}<5 \%\right)$ can be produced, but that two-body recombination processes diminish the plasma density to less than $10^{12} \mathrm{~cm}^{-3}$ in less than a microsecond.

The plasma chamber is a Pyrex tube with an inner diameter of $5 \mathrm{~cm}$. The total chamber length is $150 \mathrm{~cm}$. This size tube was chosen because it allowed a higher radio frequency power density with a $3 \mathrm{~kW}$ rf source. This power density is slightly higher than the expected power density required to sustain the discharge based on the premise that $40-60 \mathrm{eV}$ is required per electron-ion pair created. These parameters are typical ${ }^{18}$ of most low pressure discharges, but we expect that, in the high pressure regime, the electron temperature will be lower, and the energy cost per electron-ion pair created will be higher. The laser beam enters the chamber through a 2.5 $\mathrm{cm}$ diameter window mounted at one end. The window is coated for $98 \%$ transparency at $193 \mathrm{~nm}$. Gas flow valves are also located at this end to allow a variety of gases to enter the chamber.

The radio frequency source is a $13.56 \mathrm{MHz}$ single frequency generator with a maximum output power of $3000 \mathrm{~W}$ (Advanced Energy RFG3000). Power is transmitted through $50 \Omega$ cable to a capacitive matching network and to the antenna which surrounds the plasma chamber. The antenna is $\frac{1}{4}$ in. copper tubing which has been wound about a mandril. All interconnects are type- $N$ coaxial feeds. The chamber and antenna are enclosed in an aluminum stove pipe shield 8.5 $\mathrm{cm}$ in diameter. The capacitive network consists of two 
vacuum variable capacitors and is shielded from the plasma chamber by enclosing it in a conducting box.

A diaphragm pump and high-vacuum turbomolecular pump are used to evacuate the plasma chamber to a base pressure of $P \sim 10^{-7}$ Torr. A needle valve is opened to allow the working gas to enter the chamber. The TMAE purity is ensured by letting the turbomolecular pump run for several minutes with the TMAE valve open. This is an important procedure since TMAE is known to be reactive to oxygen ${ }^{19}$ over long periods. In our experiments, we filled the liquid TMAE in a stainless steel vacuum vessel. A separate valve allows argon into the system until the total pressure is raised to the appropriate level.

\section{B. Impedance measurement}

The plasma-loaded antenna impedance can be matched to the $50 \Omega$ transmission line by inserting a series capacitor $C_{1}$ and a shunt capacitor $C_{2}$ across the antenna. The capacitor values necessary to match the load impedance to the transmission line impedance are given as

$$
\begin{aligned}
& C_{1}=\frac{\left(R / R_{0}\right)}{\gamma \omega}, \\
& C_{2}=\frac{(X-\gamma)}{\left(X^{2}+R^{2}\right) \omega},
\end{aligned}
$$

where the parameter $\gamma$ is given by

$$
\gamma=\sqrt{\frac{R}{R_{0}}} \sqrt{X^{2}+R\left(R-R_{0}\right)},
$$

where $R+i X$ is the plasma-loaded antenna impedance and $R_{0}$ is the characteristic impedance of the coax feed to the matchbox.

The plasma-loaded antenna impedance is measured by matching the capacitive network/antenna combination to the $50 \Omega$ transmission line. Under matching conditions, the input impedance to the entire network is $50 \Omega$. In order to measure the plasma-loaded antenna impedance, the antenna, with its unknown impedance value $Z_{\text {ant }}=R+i X$, is disconnected from the remainder of the circuit. Under match conditions, the impedance seen by a wave traveling back toward the generator is the conjugate of $Z_{\text {ant }}, Z_{\text {ant }}^{*}=R-i X$. With the generator disconnected and replaced with a $50 \Omega$ load, the input impedance $Z_{\text {ant }}^{*}$ can be measured directly with a network analyzer. The plasma and loaded antenna impedance can then be compared with our ANTENAII code to determine the plasma density and collisionality.

\section{Plasma density measurement}

Plasma density measurements are carried out by a 105 $\mathrm{GHz}$ millimeter wave interferometer. The interferometer is located $8 \mathrm{~cm}$ axially from the end of the antenna. The distance between the transmitting horn and the plasma is $10 \mathrm{~cm}$, and the distance from the plasma to the receiving horn is 5 $\mathrm{cm}$. The line-integrated plasma density for a plasma of thickness $d$ is given as a function of the change in phase of a transmitted wave. For electron densities much less than the cutoff frequency $\left(\omega \gg \omega_{p}\right)$, the change in phase is linearly proportional to the plasma electron density,

$$
n_{e} \approx \frac{2 \phi c}{d} \frac{\omega \epsilon_{0} m_{e}}{e^{2}} .
$$

\section{PLASMA CONDITIONS}

Typically, in lower pressure conditions, the antenna can create the plasma through capacitive breakdown of the working gas due to the drop in voltage across the ends of the inductive antenna coil. The initially created electrons help to ionize more electrons in the sheath near the antenna and, after several rf cycles, the plasma goes into an inductive coupling mode. The transition between the capacitive mode and inductive mode is quite noticeable and is characterized by much stronger light emission from the plasma. ${ }^{20}$ The transition is sudden and discontinuous as described in several papers. ${ }^{21-23}$ The inductive mode is much more efficient in sustaining the plasma due to the diminishing effects of ion losses in the sheath as is well known in the plasma processing area of study. ${ }^{24,25}$

At high pressures, capacitive initial ionization is more difficult due to the higher electric fields required to break down the working gas. It is, therefore, desirable to provide the initial condition of high plasma density such that power can be transferred inductively from the antenna to the plasma without going through the capacitive mode.

The laser-produced plasma is a noninvasive way to provide a high density initial condition to which the antenna can couple power. This is accomplished by photoionization of an organic molecule, TMAE. The plasma is formed in $20 \mathrm{~ns}$ by an UV laser operating in the $193 \mathrm{~nm}(6.42 \mathrm{eV}$ per photon) argon-fluoride mode. The vertical ionization energy for TMAE is $6.11( \pm 0.02) \mathrm{eV}^{26}$ The laser has an internal feedback which adjusts cathode voltages and halogen concentrations, which limits the shot-to-shot energy variation to $\Delta E / E<5 \%$. An output energy of $200 \mathrm{~mJ}$ has been shown to be sufficient to create plasma densities of over $10^{13} \mathrm{~cm}^{-3} \cdot{ }^{10}$ The laser ionizes a small fraction of the available TMAE atoms and also produces excited states of TMAE which may give rise to further ionization of the remaining TMAE molecules after the laser is extinguished. ${ }^{27}$ The $1 / e$ photon absorption length, $\alpha$, at 60 mTorr is $48 \mathrm{~cm},{ }^{28}$ and has been verified by our previous Langmuir probe measurements in a low pressure TMAE plasma.

The initial plasma density near the laser input window is approximately $n_{e} \approx 4 \times 10^{13} \mathrm{~cm}^{-3}$ and $T_{e}=0.3 \mathrm{eV}$ as measured by the Langmuir probes ${ }^{10,11}$ in 60 mTorr of TMAE. Plasma density fluctuations along the axis of the laser beam are evident due to photon absorption. The density can be calculated by the photon flux, and can be represented by

$$
n_{e}=n_{e 0} \exp (-\alpha z) \text {. }
$$

The initial plasma density near the antenna is $n_{e} \approx 2$ $\times 10^{13} \mathrm{~cm}^{-3}$ and initial plasma densities in excess of 1 $\times 10^{13} \mathrm{~cm}^{-3}$ are measured $1 \mathrm{~m}$ from the laser window by Langmuir probe measurements in 60 mTorr of TMAE. 


\section{THEORETICAL FRAMEWORK}

\section{A. Electron density decay}

The electron density is a function of position and time, $n_{e}=n_{e}(r, t)$, and can be determined by the continuity equation. The continuity equation, including the ambipolar diffusion term, the ionization frequency term $\nu_{i z}$, and the twobody and three-body recombination terms, is

$$
\frac{\partial n}{\partial t}=D_{a} \nabla^{2} n+\nu_{i} n-\alpha_{r} n^{2}-\beta_{r} n_{g} n^{2},
$$

where $\alpha_{r}, \beta_{r}$, and $n_{g}$ are the two-body recombination rate, three-body recombination rate, and the neutral gas pressure, respectively. In most moderate pressure $(P<100$ mTorr $)$ noble gas plasmas, the main mechanism for plasma density decay is diffusion of the plasma to the chamber wall. However, it is the effective recombination process which limits the peak density obtainable for the discharge at high density and pressure.

In high pressure, $(P>100 \mathrm{mTorr})$ high density $\left(n_{e}\right.$ $>10^{12} \mathrm{~cm}^{-3}$ ) plasmas, the recombination terms play a larger role in density decay [Eq. (6)]. The two-body recombination term for argon is estimated to be $\alpha_{r}^{\mathrm{Ar}}=6.5 \times 10^{-8} \mathrm{~cm}^{3} \mathrm{~s}^{-1},{ }^{29}$ and for a plasma with a peak density of $n_{e}=4.3$ $\times 10^{12} \mathrm{~cm}^{-3}$, the number of particles lost per second due to recombination is much larger than the number lost to diffusion. The three-body recombination effect also becomes important at higher pressures. However, for the dominant threebody recombination process that involves an ion, an electron, and an argon neutral, the neutral gas pressure at which it becomes comparable to two-body recombination process can be estimated. For $\alpha_{r}^{\mathrm{Ar}}=6.5 \times 10^{-8} \mathrm{~cm}^{3} \mathrm{~s}^{-1}$ (Ref. 29) and $\beta_{r}^{\mathrm{Ar}}=10^{-26} \mathrm{~cm}^{6} \mathrm{~s}^{-1},{ }^{30}$ and using $\alpha_{r}^{\mathrm{Ar}}=\beta_{r}^{\mathrm{Ar}} n_{g}$, the $\operatorname{argon}$ pressure at which these two terms become comparable is 202 Torr. Thus for the pressure range of interest, the three-body recombination contribution is small and is negligible.

Two-body recombination has an even stronger effect in a TMAE plasma than in an argon plasma. The two-body recombination coefficient of TMAE is much larger than that of argon $\alpha_{r}^{\mathrm{TMAE}} \approx 2 \times 10^{-6} \mathrm{~cm}^{3} \mathrm{~s}^{-1}$ at $P=1$ Torr. ${ }^{10}$ The situation is much more complicated, of course, when the plasma is madeup of two ion species. For example, consider that the total electron density is equal to the sum of the TMAE ions and the argon ions. Then the source-free time-dependent density decay due to two-body recombination is given by

$$
\begin{aligned}
\frac{\partial n_{e}}{\partial t} & =-\alpha_{r}^{\mathrm{TMAE}} n_{e} n_{i}^{\mathrm{TMAE}}-\alpha_{r}^{\mathrm{Ar}} n_{e} n_{i}^{\mathrm{Ar}}, \\
& =-\alpha_{r}^{\mathrm{TMAE}} n_{e} n_{i}^{\mathrm{TMAE}}-\alpha_{r}^{\mathrm{Ar}} n_{e}\left(n_{e}-n_{i}^{\mathrm{TMAE}}\right) \\
& \approx-\alpha_{r}^{\mathrm{Ar}} n_{e}^{2}-\alpha_{r}^{\mathrm{TMAE}} n_{e} n_{i}^{\mathrm{TMAE}} .
\end{aligned}
$$

One can see that the recombination rate for the composite two ion species system is faster than it would be for pure argon by an additional factor which is proportional to the TMAE ion density. Likewise, the recombination rate for the composite species is slower than it would be for pure TMAE by a factor proportional to the argon ion density. Experimentally, there may be more than two ion species due to the

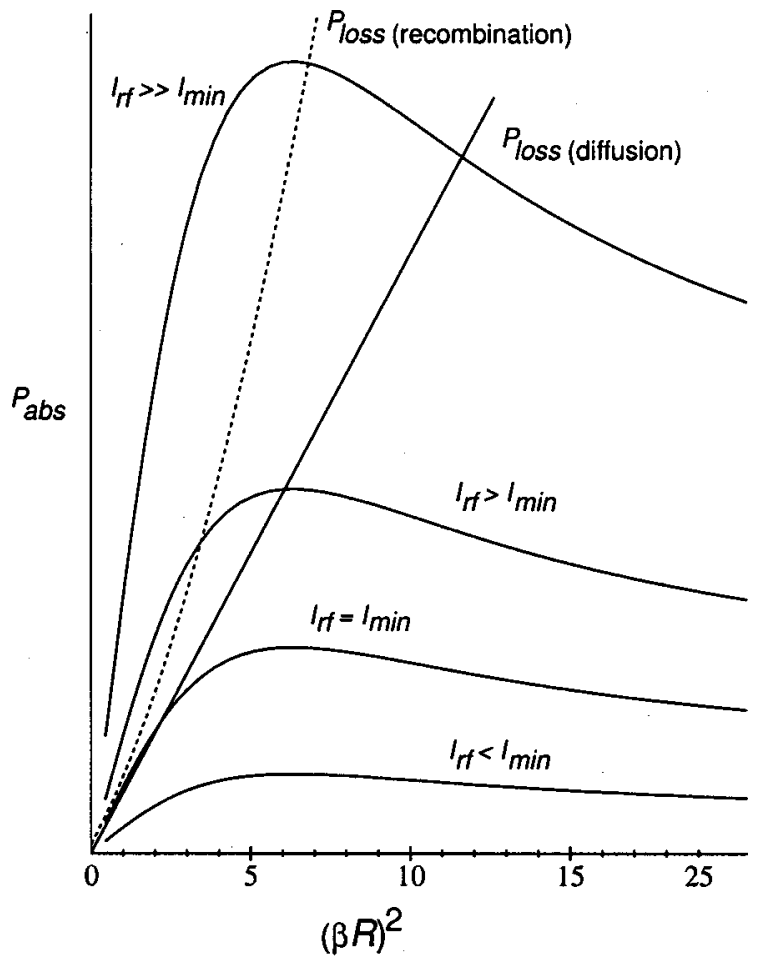

FIG. 2. Absorbed power as a function of $(\beta R)^{2}$. The two loss curves show how recombination losses, represented by the dashed line and proportional to the square of the electron density, become a larger factor than diffusion.

dissociation of TMAE. We will assume that the dominant ionizing collisions are with TMAE and argon. This is reasonable given the low ionization potential of TMAE. At these low electron temperatures, most collisions with other species will likely be either excitation or elastic collisions, so no other ion species are likely to be present.

In summary, the dominant process for electron density decay in this experiment is recombination with diffusion being a minor factor for particle loss. The effect of recombination is a faster density decay than is the case for diffusion, and this decay tends to relax density gradients that are present in the plasma. The net effect is that the core plasma density profile can be considered relatively uniform in the radial direction. This is in contrast to lower pressure, lowdensity plasmas where diffusion is the dominant mechanism for density decay and gives rise to more peaked radial density profiles.

Figure 2 illustrates a power balance curve where the dominant high pressure recombination process for our case is added in addition to the diffusion process discussed by Lieberman and Lichtenberg. ${ }^{24}$ It considers power balance in a plasma as a function of the radial wave number times the plasma radius $(\beta R)$, where $\left(\beta=\left[\omega \mu_{0} e^{2} n_{e} / m \nu\right)^{1 / 2}\right]$. Different inductive antenna coil currents $I_{\mathrm{rf}}$ are considered. Figure 2 shows the variation in the absorbed power as the density changes which is incorporated into the radial wave number $\beta$, but it also shows the loss curve for two-body recombination by the dashed line. The line representing diffusive losses is shown for comparison. At a given coil current, the steadystate plasma density can be deduced by determining at which density the curve for the absorbed power intersects the re- 


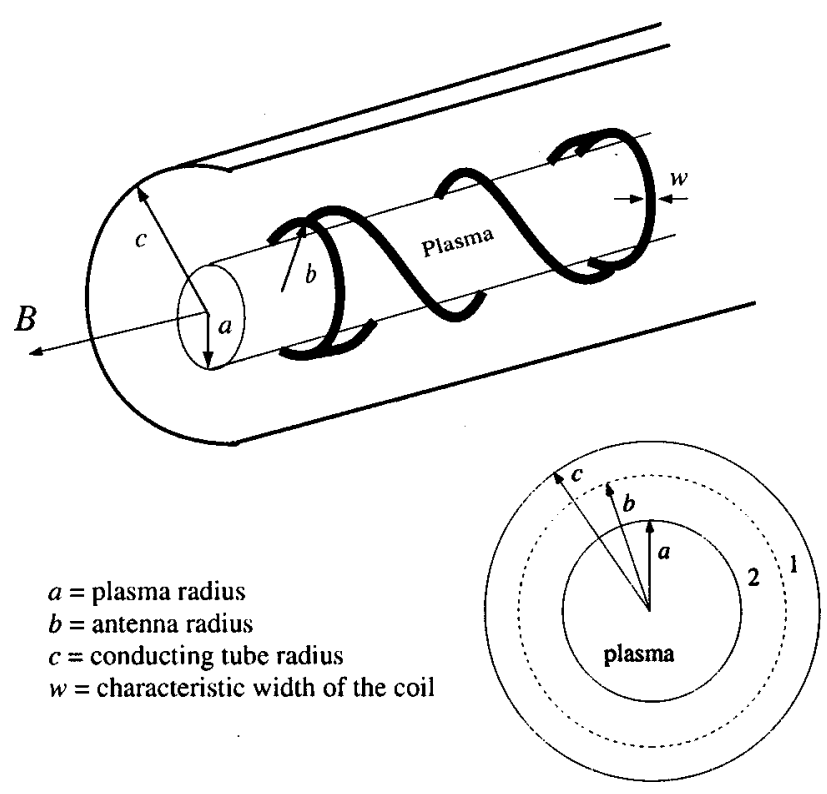

FIG. 3. Antenna-plasma geometry used for ANTENAII code calculation.

combination losses represented by the dashed line. It is easy to see that the density is nearly independent of the coil current for values of $\beta R>2.5$. The steep nature of the recombination loss curve shows how two-body recombination puts an upper bound on the density obtainable, because any increase in the absorbed power is offset by the substantial losses due to recombination.

\section{HELICAL COIL}

The power transferred to the plasma and the antenna impedance can be calculated from the ANTENAII code $^{15,16,31,32}$ The antenna-plasma geometry used in the ANTENAII code is shown in Fig. 3. This code incorporates the collisional plasma dielectric tensor as well as the antenna spectrum, radial plasma profile, and field boundary conditions. The power transferred to the plasma can be written as

$$
P_{c}=-\frac{1}{2} \int \mathbf{E} \cdot \mathbf{J}^{*} \mathrm{~d} \mathbf{V}
$$

The impedance of the antenna is then written as

$$
Z=\frac{2 P_{c}(\omega)}{|I|^{2}}=R_{\text {ant }}+i X_{\text {ant }},
$$

where $R_{\text {ant }}=$ radiation resistance of the antenna, and $X_{\text {ant }}$ $=$ reactance of the antenna.

To utilize our ANTENAII code for a helix, we approximate it with a multiturn loop since a shallow pitch helix primarily excites the transverse electric (TE) $m=0$ azimuthal modes. The pitch angle determines the magnitude of the other modes, $m \neq 0$, with the current distribution being

$$
\mathbf{J}=\mathbf{J}_{0} \cos (\psi) \hat{\theta}+\mathbf{J}_{0} \sin (\psi) \hat{z},
$$

where $\psi$ is the pitch angle of the helix. For a tightly wound helix, the pitch angle is limited by the characteristic width of the coil strapping, $w$, by $\psi>w / 2 \pi R$. Since we are using a coil with a small characteristic width of $0.65 \mathrm{~cm}$, and our
TABLE I. Radiation resistance and reactance of a multiturn helix with a coil spacing of $1 \mathrm{~cm}$ calculated by ANTENAII for a uniform argon plasma $2.5 \mathrm{~cm}$ in radius with a density of $n_{e}=4.2 \times 10^{12} \mathrm{~cm}^{-3}$ at a pressure of 1 Torr.

\begin{tabular}{ccc}
\hline \hline Turns & $\begin{array}{c}R_{\text {ant }} \\
(\Omega)\end{array}$ & $\begin{array}{c}X_{\text {ant }} \\
(\Omega)\end{array}$ \\
\hline 1 & 0.5 & 5.3 \\
2 & 1.6 & 13.0 \\
3 & 2.9 & 21.0 \\
4 & 4.1 & 28.0 \\
5 & 6.9 & 49.0 \\
\hline \hline
\end{tabular}

chamber radius is relatively large at $2.5 \mathrm{~cm}$, we can reasonably assume that the current density is entirely in the azimuthal direction. The helix is also an attractive antenna since the anticipated low plasma radiation resistance at high pressures will be enhanced by the square of the number of turns.

The limiting approximation in a closely spaced $n$-turn helix plasma load is the absence of magnetic flux leakage between successive coils and a uniform magnetic field in the axial direction. In order to simulate the idealized situation, a very narrow coil spacing is needed, however, experimentally there is a lower limit to the spacing. If the coil is wound too tightly, then the voltage potential difference between successive coil loops becomes so large that arcing occurs between the loops. Table I shows the relationship as calculated by ANTENAII for a tightly wound coil, where the coil spacing is $3.0 \mathrm{~cm}$ and the characteristic thickness $w$ is $0.65 \mathrm{~cm}$. The coil has a radius of $b=3.0 \mathrm{~cm}$ and surrounds a plasma with radius of $a=2.5 \mathrm{~cm}$ with a density of $4.2 \times 10^{12} \mathrm{~cm}^{-3}$, and the pressure is 1 Torr. ANTENAII code calculations confirm that the square law is quite valid if one compares the four-turn and the five-turn coils where the magnetic field uniformity would be a good approximation.

The pitch angle is approximated in the code simulation by appropriate spacing of the loops. Figure 4 shows the dependence of the radiation resistance on the coil spacing for a coil with a width of $0.65 \mathrm{~cm}$ and coil radius $b=3.0 \mathrm{~cm}$ which surrounds a plasma of radius $a=2.5 \mathrm{~cm}$ with a density of

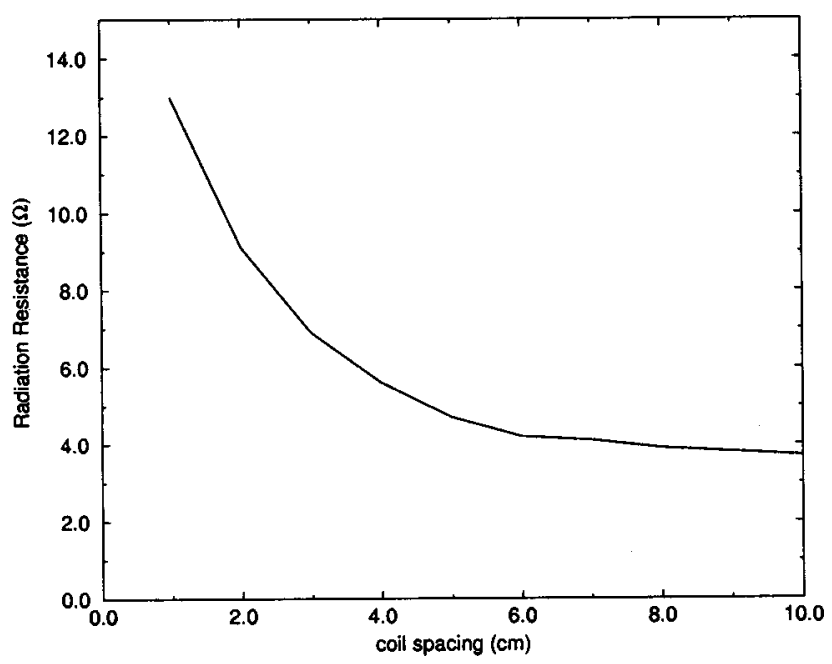

FIG. 4. Antenna radiation resistance vs coil spacing of a five-turn helical coil calculated by ANTENAII. 

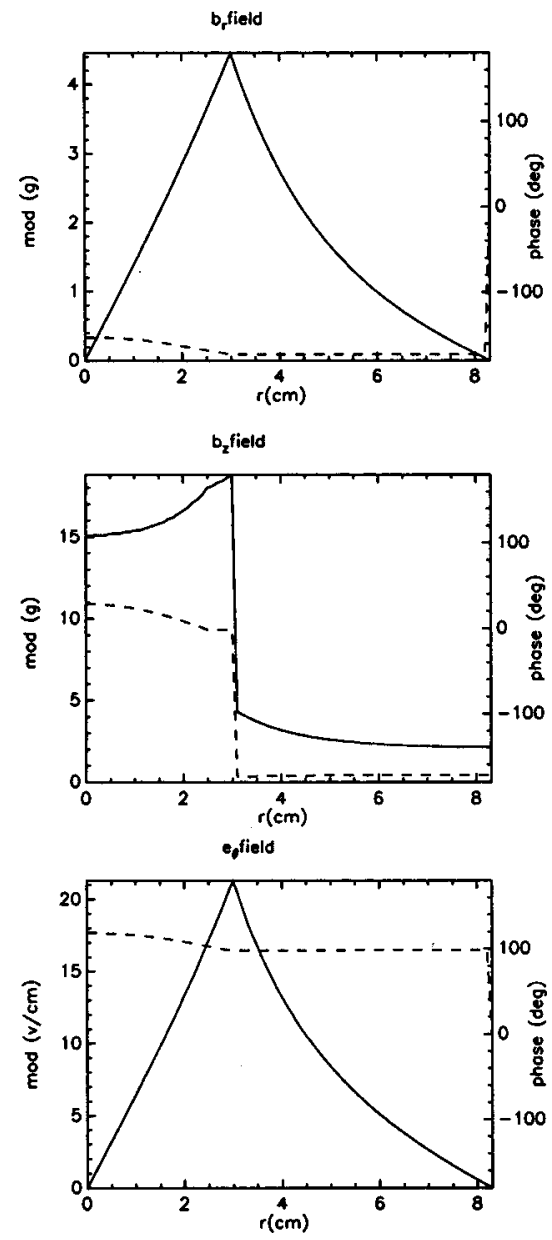

FIG. 5. Radial variation in field quantities $B_{r}, B_{z}$, and $E_{\theta}$ at the midplane of a tightly wound five-turn helical coil antenna where the interloop coil spacing is $3 \mathrm{~cm}$.

$4.2 \times 10^{12} \mathrm{~cm}^{-3}$, at a pressure of 1 Torr. In general, a very tightly wound coil with a very small pitch angle will be a better coupling element than a loosely wound coil with larger pitch angle. As the coil is wound tighter however, the inductance of the antenna also increases. The difficulties associated with this increased inductance are twofold. First, the higher inductance causes an increasingly larger rf voltage drop across the antenna terminals. Second, that voltage drop occurs over a shorter distance thereby increasing the magnitude of the capacitive of field. Figure 5 shows the radial variation of the field quantities for a five-turn helical coil at the center location of the antenna. The plasma radius is $a$ $=2.5 \mathrm{~cm}$ with a density of $n_{e}=4.2 \times 10^{12} \mathrm{~cm}^{-3}$ at a pressure of 1 Torr. The antenna has a radius of $b=3.0 \mathrm{~cm}$ with a characteristic width of $w=0.65 \mathrm{~cm}$, and the conducting shield has a radius of $c=8.3 \mathrm{~cm}$. Figure 6 shows the power absorbed as a function of the radius. Our studies show ${ }^{33}$ that a five-turn helical antenna ionizes and absorbs much more power for the same plasma than a Nagoya type-III coil ${ }^{34}$ for the same plasma conditions.

\section{EXPERIMENTAL RESULTS}

The experiment was carried out with the goal of determining whether a plasma could be initiated by a laser and total power absorbed

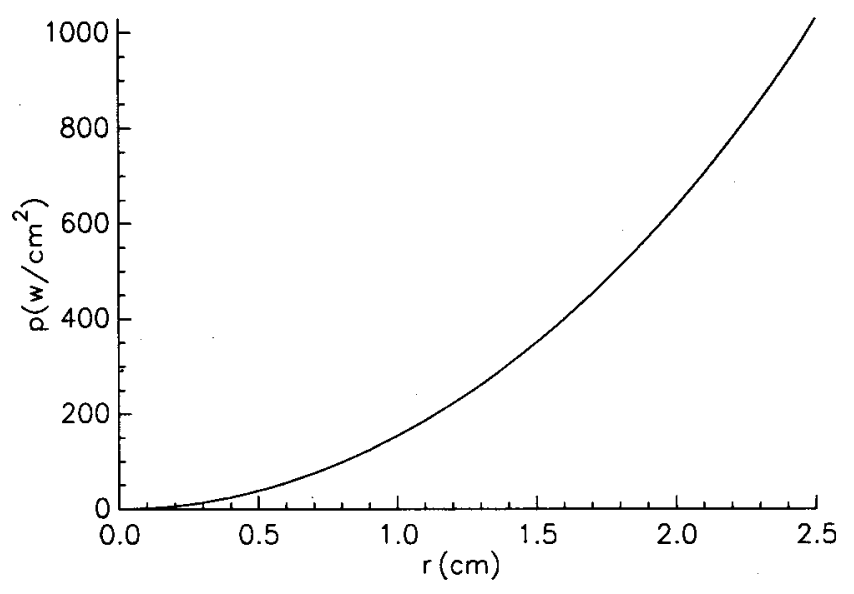

FIG. 6. Radial power deposition profile of the five-turn helical coil antenna that surrounds an argon plasma with a uniform density of $n_{e}=4.2$ $\times 10^{12} \mathrm{~cm}^{-3}$, at a pressure of 1 Torr, with a coil current of $60 \mathrm{~A}$.

then maintained at a higher pressure by inductive coupling of radio frequency power. The five-turn helical antenna was designed with the help of the ANTENAII code. When determining the length and coil spacing of the helical coil, the fiveturn helix with a length of $13 \mathrm{~cm}$ was chosen because it provided an inductance which could be easily matched with available capacitors.

The plasma was started at relatively low argon pressure, and the pressure and input rf power was increased until the plasma could not be initiated by the rf antenna alone at 80 Torr. At this point, a small amount of TMAE (2-6 mTorr) was introduced in order that the plasma could be ignited with the ultraviolet laser. The neutral argon pressure was again increased until the plasma could not be initiated with the laser and maintained with the rf power. The plasma density was measured in each case as a function of the neutral pressure of the working gas. Plasma density measurements were taken using a $105 \mathrm{GHz}$ millimeter wave interferometer.

The antenna impedance measurements were taken using a network analyzer with a Smith chart output. For typical operating parameters of the five-turn helical coil, the correction to the measured value of the antenna impedance due to the two-wire line feeds is $\Delta Z_{\text {ant }}=0.1-i 30 \Omega$.

\section{A. Five-turn helical coil}

The most effective antenna for initiating and maintaining the plasma in this experiment is the five-turn helical coil, which primarily excites the $m=0$ TE mode of field distribution. The inductive coil consists of five turns of $\frac{1}{4}$ in. copper tubing wound tightly around the plasma chamber. The inner radius of the coil is $3.0 \mathrm{~cm}$ and the total length of the antenna is $13 \mathrm{~cm}$. The vacuum impedance of the antenna was measured with the network analyzer to be $Z_{\text {ant }}=0.02+i 72 \Omega$, indicating that the vacuum reactance of this antenna is approximately five times higher than a comparable type-III antenna. ${ }^{34}$

The experiment is carried out to determine at what maximum pressure the discharge can be initiated with the capaci- 


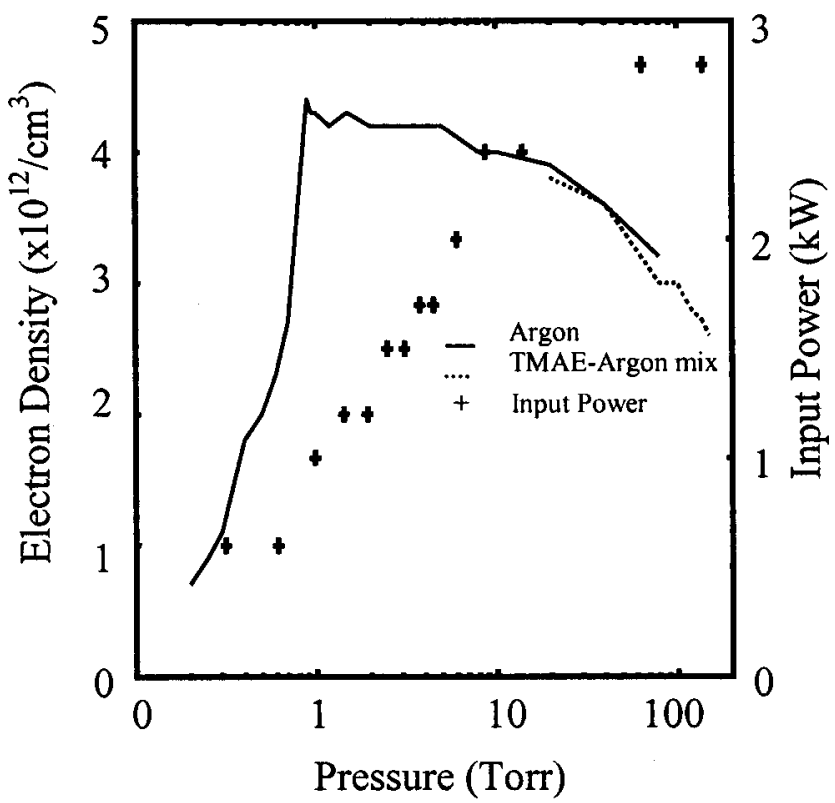

FIG. 7. Plasma density vs pressure of the five-turn helical antenna in pure argon (solid line) and in a mixture of 2-8 mTorr TMAE in argon (dashed line). The electron line-average density measurements were taken by a 105 $\mathrm{GHz}$ millimeter wave interferometer.

tive rf fields alone. The argon pressure is varied from 200 mTorr to 80 Torr. Above 80 Torr, the rf fields are not strong enough to initiate the discharge at the maximum output power of $2.8 \mathrm{~kW}$ of the generator. The voltage required to breakdown a gas is given by Paschen's law, and is proportional to the neutral gas pressure. ${ }^{35}$

The plasma density a distance of $10 \mathrm{~cm}$ from the antenna end as a function of the argon pressure is plotted as the solid line in Fig. 7 over this pressure range. Note that this measurement is a lower bound for the density under the antenna. This spacing was required to prevent rf pickup or arcing between the helix and the antenna horns. It can be seen that the plasma density is relatively constant for pressures over 1 Torr, while the collisionality is increased by two orders of magnitude as the pressure is increased from 1 to 80 Torr and the coupled rf power is increased by a factor of 4 . The peak radiation resistance, measured by the network analyzer, is for the 1 Torr case. The total antenna impedance for this case is $Z_{\text {ant }}=7.6+i 45 \Omega$. ANTENAII predicts a value of $Z_{\text {ant }}=5.4$ $+i 38 \Omega$. The maximum power absorbed occurs when $\beta R$ $\approx 2.5{ }^{24}$ The value of $\beta R$ in this experimental case is reduced by approximately an order of magnitude as the pressure is increased from 1 to 80 Torr.

There is a minimum value of $\beta R$ at which the discharge can be maintained. For every value of current, $\lim _{\beta R \rightarrow 0} P_{\text {abs }}=0$, from Fig. 2. Meanwhile, $P_{\text {loss }}$ in practice never goes to zero because there are always ohmic losses in the antenna. The discharge cannot be maintained when the ohmic losses in the antenna are greater than the radiation resistance of the antenna $\left(R_{\text {loss }}>R_{r}\right)$. According to our ANTENAII calculations, an estimate of the maximum pressure at which this antenna can maintain the discharge is about 500 Torr since the radiation resistance is $0.02 \Omega$ and below the

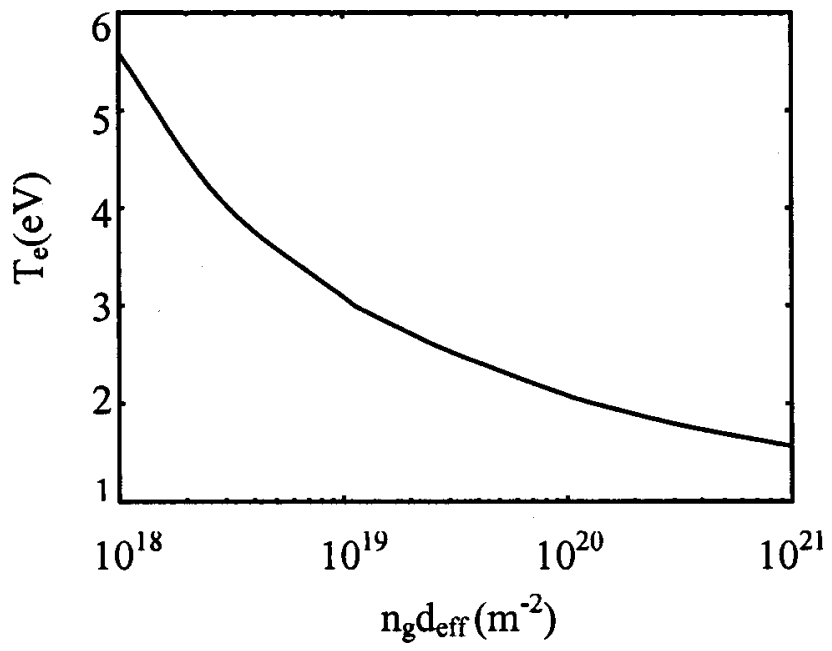

FIG. 8. $T_{e}$ vs $n_{g} d_{\text {eff }}$ of Maxwellian electrons in argon assuming loss due to diffusion to the chamber wall. The parameter $n_{g}$ is the neutral gas density, and $d_{\text {eff }}$ is an effective plasma size which takes into account the ratio of the volume to surface area as well as the sheath size.

antenna ohmic losses of $0.05 \Omega$ for this pressure at a density of $n_{e}=4 \times 10^{12} \mathrm{~cm}^{-3}$.

A theoretical model has been developed to estimate the electron temperature using a particle balance of diffusive losses and ionizing sources. ${ }^{24}$ Figure 8 shows the electron temperature as a function of the neutral pressure for an argon plasma of a given size. The parameter $n_{g} d_{\text {eff }}$ is an effective plasma size which takes into account the neutral gas pressure and sheath effects. The number of particles which diffuse to the sheath at the Bohm velocity, $u_{B}\left(T_{e}\right)$ must balance the number of particles created in the volume, which is proportional to the rate constant for ionization, $K_{i z}\left(T_{e}\right)$. The rate constants are shown in Fig. $9^{18,24}$ as a function of electron energy. The relationship between these parameters is given by

$$
n_{e} u_{B} S=K_{i z} n_{g} n_{e} V,
$$

where $S$ is approximately the surface area of the plasma inside the volume $V$. In practice, there is a small modification

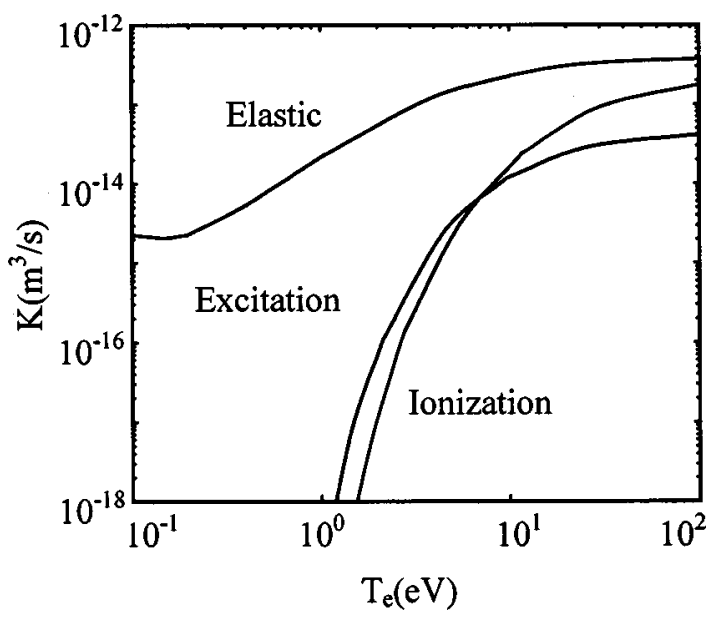

FIG. 9. Theoretical elastic, excitational, and ionizing collisional rate constants vs $T_{e}$ of argon gas. 
to $S$ in that the density has an assumed radial and axial profile, and the density at the sheath edge is not exactly $n_{e}$. For the purposes of this analysis, we assume that the gradient at the plasma edge resembles a step profile and that $S=2 \pi R^{2}$ $+2 \pi R l$ and the ratio of the volume to the size is $d_{\text {eff }}=V / S$. According to this relationship, the ratio $K_{i z} / u_{B}$ is inversely proportional to the size parameter, $n_{g} d_{\text {eff }}$, of the plasma. From this relationship, the electron temperature can be estimated from Fig. 8.

When two-body recombination is the dominant loss mechanism, as it is in this experiment, the situation changes. Now the ionization rate is not balanced by diffusive losses (proportional to $n_{e}$ ), but by recombination losses (proportional to $n_{e}^{2}$ ). The new power balance corresponding to Eq. (11) is

$$
\alpha_{r} n_{e}^{2} V=K_{i z} n_{g} n_{e} V .
$$

Since the volume $V$ appears on both sides of this equation, the plasma dimensions do not a effect the particle balance, but it is evident that the normalized rate constant is still inversely proportional to the neutral gas pressure, $K_{i z} / \alpha_{r} n_{e}$ $=1 / n_{g}$. This implies that $u_{B} / d_{\text {eff }}<\alpha_{r} n_{e}$ if two-body recombination is larger than diffusion. This means that at a given pressure, the effective plasma size is larger if recombination is the main loss mechanism than if diffusion is the main loss mechanism. Thus Fig. 8 provides an upper bound to the electron temperatures at a given neutral pressure $n_{g}$. Defining a new plasma size factor obtained from the previous considerations,

$$
d_{\mathrm{eff}}^{\prime}=u_{B} / \alpha_{r} n_{e},
$$

and utilizing the plasma parameters $\alpha_{r}=6.5 \times 10^{-8} \mathrm{~cm}^{3} \mathrm{~s}^{-1}$ and $n_{e}=4 \times 10^{12} \mathrm{~cm}^{-3}$ measured in the lab at a pressure of 1 Torr, we obtain an iterative self-consistent electron temperature estimate from Fig. 8 of $1.6 \mathrm{eV}$.

At pressures above 80 Torr, the plasma can be initiated by adding a small amount of TMAE and using the laser for breakdown. The amount of TMAE required is critical to the operation of the source and is discussed in Sec. V B. It was experimentally determined that with 2-8 mTorr of TMAE in argon, the plasma can be consistently sustained at pressures of up to 150 Torr with $2.8 \mathrm{~kW}$ of rf power. The lineintegrated plasma density, measured with the interferometer, is shown as the dotted line in Fig. 7. The measured antenna impedance under matched conditions at 150 Torr is obtained as $Z_{\text {ant }}=0.45+i 72 \Omega$ which shows how the antenna radiation resistance due to the plasma loading is reduced at high pressures.

\section{B. TMAE neutral pressure}

The amount of TMAE which is required to initiate the plasma is a critical issue. Too much TMAE causes the plasma to recombine too quickly and increases electron energy loss due to inelastic collisions with a polyatomic molecule. It also does not allow sufficient axial extent of the laser-initiated high density plasma into the radio frequency coil region due to strong laser photon absorption by the TMAE molecules. Conversely, too little TMAE will not pro-

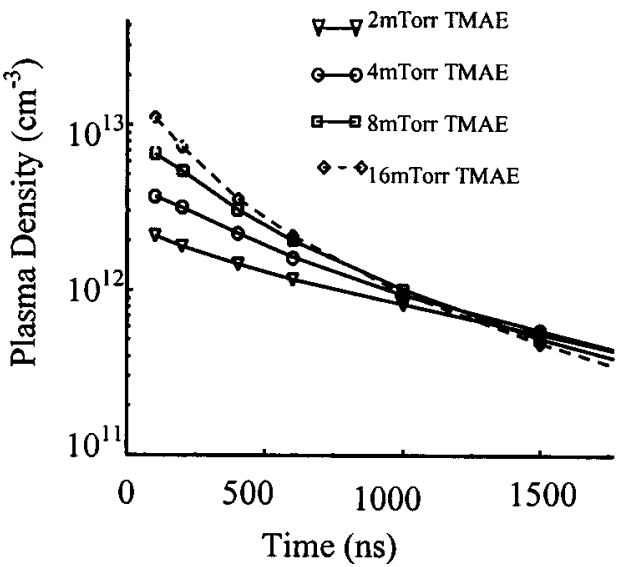

FIG. 10. Plasma density as a function of time for several filling pressures of TMAE. The density measurement was taken by a Langmuir probe inserted into the laser-produced plasma. The axial position of the probe is $10 \mathrm{~cm}$ from the laser input window.

vide enough electrons to initiate the plasma. An experiment was carried out that pertained to the laser-produced plasma electron density as a function of the TMAE neutral pressure. The plasma density as a function of time without rf sustainment is plotted in Fig. 10 for four neutral TMAE pressures without argon present. These density measurements ${ }^{12}$ were taken with a Langmuir probe approximately $10 \mathrm{~cm}$ from the laser input window, and show that about 2 mTorr of TMAE is required to initiate a plasma with a density of greater than $10^{12} \mathrm{~cm}^{-3}$.

Lab tests showed that if more than 20 mTorr of TMAE is used, the discharge cannot be maintained at 1 Torr argon pressure with $2 \mathrm{~kW}$ of $\mathrm{rf}$ due to the fast recombination rate and the exponential axial decay from the UV window of the laser-initiated plasma. Using the five-turn helical coil, the plasma density versus TMAE neutral pressure at a fixed rf power of $2 \mathrm{~kW}$ is noted in Table II. The total gas pressure is 1 Torr, with the background gas being argon. The plasma density in the 2 mTorr TMAE case is very nearly equal to the plasma density in the pure argon case, and increasing the TMAE pressure causes a subsequent decrease in the rfsustained plasma density. In the case of 60 mTorr of TMAE, it was not possible to maintain the discharge at 1 Torr, so an argon pressure of 600 mTorr was used, further reducing the resultant density.

The data suggest that while TMAE could be considered a good initiator of a high-pressure discharge, its recombination rate limits its usefulness for rf sustainment. One could

TABLE II. TMAE pressure and electron density variations in high pressure (1 Torr) argon plasmas.

\begin{tabular}{ccc}
\hline \hline $\begin{array}{c}\text { TMAE base pressure } \\
(\mathrm{mTorr})\end{array}$ & $\begin{array}{c}n_{e} \\
\left(10^{12} \mathrm{~cm}^{-3}\right)\end{array}$ & $\begin{array}{c}\text { Total pressure } \\
\text { (argon in Torr) }\end{array}$ \\
\hline 2 & 4.0 & 1 \\
5 & 3.0 & 1 \\
10 & 2.9 & 1 \\
20 & 2.7 & 1 \\
60 & 1.3 & 0.6 \\
\hline \hline
\end{tabular}




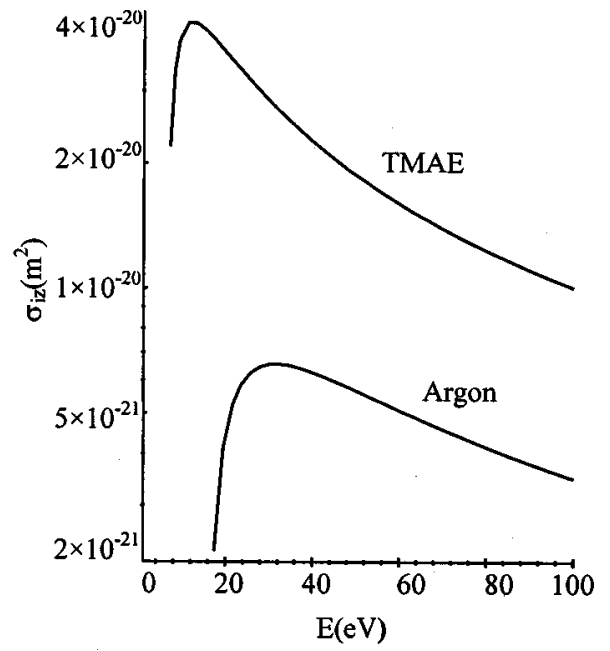

FIG. 11. Classical Thompson ionization cross section of TMAE and argon molecules as a function of the electron energy.

use a small amount of TMAE to initiate the plasma discharge with an UV flash tube, but the main working gas needs to have a much longer electron lifetime than TMAE. This could be accomplished by initiating the plasma with TMAE then utilizing the electrons to further sustain the plasma by radio frequency power and flowing the majority gas through the system to displace the TMAE.

One interesting detail of the TMAE pressure dependance depicted in Table II is that the density falls off quite steeply with an increase in TMAE pressure, and that the discharge is more difficult to maintain in the inductive mode when there is more TMAE present. The explanation for this lies in the fact that the recombination is so fast with higher TMAE concentrations that the average electron does not have time to make another ionizing collision before it is lost to twobody recombination. It is important, as shown in the following analysis, that there is a specific fraction of TMAE molecules which should be ionized in order to maximize the density in the argon/TMAE mixture. First, we will assume that the maximum density with radio frequency sustainment at a given pressure can be obtained by using a pure argon discharge. This maximum density is limited by two-body recombination processes. By adding a small amount of TMAE, that maximum density can also be maintained in the argon/ TMAE mixture. The optimal TMAE pressure can be estimated by using the Thompson model for the ionization collision cross section for TMAE and argon.

The classical Thompson ionization cross section ${ }^{36}$ for ionization is given by

$$
\sigma_{i z}=\pi\left(\frac{e}{4 \pi \epsilon_{0}}\right)^{2} \frac{1}{E}\left(\frac{1}{E_{i z}}-\frac{1}{E}\right) .
$$

Figure 11 shows the theoretical Thompson ionization cross section for both TMAE $\left(E_{i z}^{\mathrm{TMAE}}=6.1 \mathrm{eV}\right)$ and $\operatorname{argon}\left(E_{i z}^{\mathrm{Ar}}\right.$ $=15.8 \mathrm{eV}$ ). To calculate the rate constants, $K_{i z}$, Eq. (14) needs to be integrated over the energy distribution,

$$
K_{i z}=\int_{0}^{\infty} \sigma_{i z} f(E) d E
$$

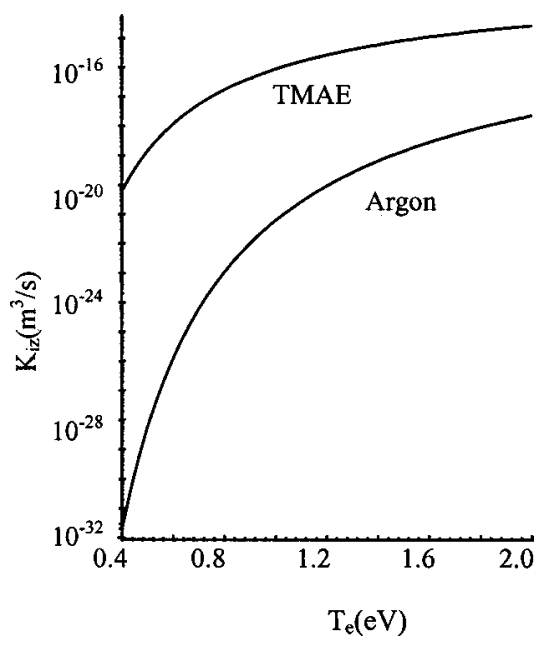

FIG. 12. Rate constants $K_{i z}$ of ionizing collisions for argon and TMAE as a function of electron temperature $T_{e}$.

where $f(E)$ is the electron energy distribution function. Only the high energy electrons in the tail of the distribution, along with electrons close to the ionization threshold, contribute significantly to $K_{i z}$. For an assumed Maxwellian energy distribution where the estimated value of the electron temperature $\left(T_{e}=1.6 \mathrm{eV}\right)$ is much less than the threshold energy for ionization $\left(T_{e} \ll E_{i z}^{\mathrm{Ar}}, E_{i z}^{\mathrm{TMAE}}\right)$, we can expand $f(E)$ about the maximum cross section,

$$
\sigma_{i z}(\max )=\frac{\pi}{4}\left(\frac{e}{4 \pi \epsilon_{0}}\right)^{2} \frac{1}{E_{i z}^{2}},
$$

to approximate the rate constant. The expansion about $\sigma_{i z}(\max )$ is

$$
\sigma_{i z}=\sigma_{i z}(\max ) \frac{E-E_{i z}}{E_{i z}} .
$$

An estimate of the rate constants can be found by integrating Eq. (17) over the electron energy distribution, giving

$$
K_{i z}=\sigma_{i z}(\max ) \bar{v}\left(1+\frac{2 T_{e}}{E_{i z}}\right) \exp \left(-E_{i z} / T_{e}\right),
$$

where $\bar{v}=\sqrt{8 e T_{e} / \pi m}$ is the average electron velocity. Figure 12 shows the functional behavior of the ionization rate constants on the electron temperature. The frequency of ionization is given as $\nu_{i z}=K_{i z} n_{g}$, where $n_{g}$ is the neutral gas density of each species.

The two-body recombination coefficient of TMAE is larger than the two-body recombination coefficient of argon. However, the ionization rate constant is also larger for TMAE than for argon, in order to maintain the high plasma density. Either the partial pressure of TMAE needs be low enough that there are more ionizing collisions with argon than with TMAE, or a particularly small fraction of the TMAE molecules should be ionized in order to keep the recombination rate $\partial n / \partial t=-\alpha_{r}^{\mathrm{TMAE}} n_{e}^{2}$ small. At a pressure of 40 Torr, and a density of $n_{e}=3.6 \times 10^{12} \mathrm{~cm}^{-3}$, the positive and negative effects of using TMAE balance out (see Fig. 7) since the density is the same with pure argon as it is 
with 2-8 mTorr TMAE seeded in 40 Torr argon. From power balance considerations, in pure argon at high pressure where $\alpha_{r}^{\text {argon }}=6.5 \times 10^{-8} \mathrm{~cm}^{3} / \mathrm{s},{ }^{29}$

$$
K_{i z}^{\text {argon }} n_{g}^{\text {argon }} n_{e}=\alpha_{r}^{\text {argon }} n_{i} n_{e}
$$

yields $K_{i z}=1.7 \times 10^{-19} \mathrm{~m}^{3} / \mathrm{s}$. This is consistent with an electron temperature of $T_{e}=1.6 \mathrm{eV}$ when referring to the ionization rate constant for argon in Fig. 12.

For an electron temperature of $T_{e}=1.6 \mathrm{eV}$, the rate constant for ionizing collisions of TMAE is $K_{i z}=1$ $\times 10^{-15} \mathrm{~m}^{3} / \mathrm{s}$ from Fig. 12. If we add TMAE to the gas mix, the compound two-component plasma can be described by power balance with

$$
\begin{aligned}
K_{i z}^{\text {argon }} & n_{g}^{\text {argon }} n_{e}+K_{i z}^{\mathrm{TMAE}_{g}} n_{g}^{\mathrm{TMAE}} n_{e} \\
= & \alpha_{r}^{\text {argon }} n_{i}^{\text {argon }} n_{e}+\alpha_{r}^{\mathrm{TMAE}} n_{i}^{\mathrm{TMAE}} n_{e} .
\end{aligned}
$$

If we assume that $n_{i}^{\text {argon }}+n_{i}^{\mathrm{TMAE}}=n_{e}$, which is valid for singly ionized plasmas,

$$
\begin{aligned}
K_{i z}^{\text {argon }} & n_{g}^{\text {argon }}+K_{i z}^{\text {TMAE }} n_{g}^{\text {TMAE }} \\
& =\alpha_{r}^{\text {argon }}\left(n_{e}-n_{i}^{\mathrm{TMAE}}\right)+\alpha_{r}^{\mathrm{TMAE}} n_{i}^{\mathrm{TMAE}},
\end{aligned}
$$

and substituting Eq. (19) since the electron densities for pure argon and the TMAE/argon mix are equal,

$$
K_{i z}^{\mathrm{TMAE}} n_{g}^{\mathrm{TMAE}}=-\alpha_{r}^{\mathrm{argon}} n_{i}^{\mathrm{TMAE}}+\alpha_{r}^{\mathrm{TMAE}} n_{i}^{\mathrm{TMAE}} .
$$

We utilize the condition that $\alpha_{r}^{\mathrm{TMAE}} \gg \alpha_{r}^{\text {argon }}$ and rearrange the terms to find the fractional ionization of TMAE molecules at $T_{e}=1.6 \mathrm{eV}$,

$$
\frac{n_{i}^{\mathrm{TMAE}}}{n_{g}^{\mathrm{TMAE}}}=\frac{K_{i z}^{\mathrm{TMAE}}}{\alpha_{r}^{\mathrm{TMAE}}} \approx 1.5 \times 10^{-2} .
$$

Experimentally, we find that 2 mTorr of TMAE in 1 Torr argon is a high enough TMAE partial pressure for the laser to ionize a sufficient number of TMAE molecules in order to inductively couple rf power to the plasma. Any additional TMAE above 6 mTorr tended to decrease the plasma density due to the effects of TMAE two-body recombination. At 7 mTorr of TMAE in 1 Torr argon, the neutral TMAE concentration is $n_{g}^{\mathrm{TMAE}}=2.3 \times 10^{14} \mathrm{~cm}^{-3}$, meaning a $1.5 \%$ fractional ionization yields a plasma density of $n_{i}^{\mathrm{TMAE}}=3.4$ $\times 10^{12} \mathrm{~cm}^{-3}$. This implies there are likely very few argon ions when 7 mTorr TMAE is added to the gas mixture. The number of argon ions present at TMAE partial pressures below 7 mTorr provide some ions which have longer lifetimes than TMAE ions.

The relative role of TMAE and argon ionization can be seen by examining Fig. 12. If the neutral pressure is increased, the electron temperature is decreased, but at an electron temperature of $1.6 \mathrm{eV}$, the corresponding decrease in the TMAE ionization rate constant is much less than the corresponding decrease in the argon rate constant. TMAE allows us to decrease the electron temperature without significantly reducing the ionization rate constant as long as we are ionizing mainly TMAE molecules. As a result, the pressure at which the seeded plasma can be sustained with our $2.8 \mathrm{~kW}$ source is higher than the pressure at which the discharge could be sustained with argon alone.
TABLE III. Power requirements for sustaining high density discharge at several neutral pressures of argon and estimated energy required per ionelectron pair created in argon.

\begin{tabular}{ccc}
\hline $\begin{array}{c}\text { Pressure } \\
(\text { Torr })\end{array}$ & $\begin{array}{c}\text { Power } \\
(\mathrm{kW})\end{array}$ & $\begin{array}{c}E_{c} \text { per electron ion } \\
(\mathrm{eV})\end{array}$ \\
\hline 1.0 & 1.7 & 29 \\
1.2 & 2.0 & 36 \\
2.0 & 2.4 & 43 \\
5.0 & 2.8 & 51 \\
8.0 & 2.8 & 56 \\
10 & 2.8 & 56 \\
20 & 2.8 & 59 \\
40 & 2.8 & 69 \\
80 & 2.8 & 87 \\
\hline \hline
\end{tabular}

TMAE can be utilized as a seed in high-pressure plasma production. The maximum pressure achieved was positively affected by the addition of small amounts of TMAE which are ionized by the laser (or UV flash tube) and provide substantial electron density. This allows a lower power radio frequency source which can sustain the plasma.

\section{Power requirements}

The power requirements for sustaining the discharge can be estimated by calculating the electrons lost due to twobody recombination. For a density experimentally measured at a given pressure, and a two-body recombination coefficient for argon of $\alpha_{r}=6.5 \times 10^{-8} \mathrm{~cm}^{3} / \mathrm{s},{ }^{29}$ the power required to maintain the discharge in a volume of $300 \mathrm{~cm}^{3}$ can be calculated by the volumetric power absorption. The number of particles lost due to recombination in a volume $V$ is given by

$$
N_{\text {lost }}=\int_{V} \frac{\partial n_{e}}{\partial t} d V=\int_{V}-\alpha_{r} n_{e}^{2} d V .
$$

Calculating the rf power absorbed in the plasma divided by the number of particles lost is a measurement of the energy cost per electron-ion pair created.

Experimentally, the power requirements for sustaining a pure argon plasma are displayed in Table III along with the estimated energy required per electron-ion pair based on the rf power delivered for an assumed constant electron temperature of $1.6 \mathrm{eV}$. The energy requirements as a function of the neutral pressure calculated by Eq. (24) are displayed in Fig. 13. They illustrate the higher $\mathrm{rf}$ power requirements needed to sustain argon plasmas at higher pressures in the $10^{12} \mathrm{~cm}^{-3}$ line-average density range.

\section{SUMMARY}

An organic seed gas was successfully photoionized by an ultraviolet laser, and the laser-produced plasma could be sustained by less than $2.8 \mathrm{~kW}$ of $13.56 \mathrm{MHz}$ rf power at pressures of up to 150 Torr in a volume of $300 \mathrm{~cm}^{3}$. The range of neutral pressures for which it is possible to capacitively break down the working gas is limited by the voltage potential across the ends of the antenna. The laser-produced plasma provides a high density, large volume plasma in a 


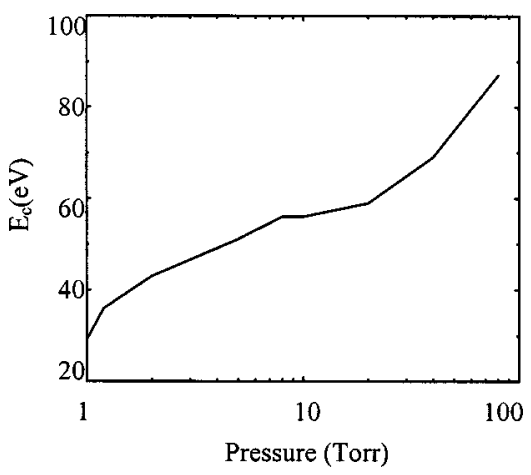

FIG. 13. Energy required per electron-ion pair created in argon plasma as a function of the argon filling pressure.

noninvasive manner that allows inductive radio frequency operation at pressures that would require substantially higher rf voltages if the plasma were ignited with the capacitive rf fields alone.

The laser-produced plasma can be sustained at lineaverage densities of $n_{e} \approx 4 \times 10^{12} \mathrm{~cm}^{-3}$ with large volumes of $300 \mathrm{~cm}^{3}$ by absorption of $13.56 \mathrm{MHz}$ radio frequency power in the plasma at $2.8 \mathrm{~kW}$ power levels. This occurs via inductive coupling of the rf fields to the plasma and collisional heating of the electrons. The currents inside the plasma are driven by electric fields which are determined by the antenna and plasma, and are modeled by the ANTENAII code. All field quantities can be described by an infinite Fourier series of wave modes which are excited by a Fourier sum of current distribution on the rf antenna. The five-turn helical coil has an azimuthally symmetric current distribution and excites the $m=0$ transverse electric wave mode. The $m=0$ transverse electric mode is found to be the most conducive for efficiently absorbing large amounts of $\mathrm{rf}$ power in the core of the plasma at these high pressures. The plasma response to the imposed electromagnetic fields can be described by a complex dielectric tensor that includes the effects of momentum-exchange collisions and the inertial effects of finite electron mass.

The maximum pressure at which the laser-initiated seed plasma could be sustained with the helical coil is limited to 150 Torr by the rf power available and energy loss requirements per electron-ion pair created. As the pressure increases, the electron temperature decreases, and the efficiency of the source is diminished due to an increase in the collisional energy lost per electron-ion pair created. This energy loss is due to the nonionizing collisions associated with the discharge including elastic collisions, collisions of excitation, and, for plasmas with polyatomic molecules, dissociating collisions, and those collisions which excite rotational and vibrational degrees of freedom.

The use of this seed gas shows promise when the plasma is operated at even higher pressures. With the correct antenna configuration, and sufficient power, the plasma needs only to have an initial condition of sufficient electron density in order to inductively couple power to the plasma. That initial condition can be satisfied by photoionization of TMAE.

The benefits of using TMAE in the steady state are limited by recombination. The TMAE ions recombine with elec- trons at a rate about 100 times faster than argon ions recombine with electrons. For this reason, there is an optimal fractional ionization of neutral TMAE molecules. If the TMAE partial pressure is too high, the fractional ionization of TMAE goes down, and the inelastic collisions which result absorb so much power that the discharge cannot be maintained. If the TMAE partial pressure is too low, the discharge can still be maintained, but the viability of initiating the discharge using the laser decreases due to the fact that the argon molecule is not photoionized by the $193 \mathrm{~nm}$ UV laser. TMAE provides a molecule which interacts quite favorably with the UV laser, and gives a high density initial condition which allows efficient coupling of $\mathrm{rf}$ power to the plasma. An optimum scenario would be one in which one pulse injects the seed TMAE and fast firing of a laser or UV flash tube initiates the plasma. Strong gas flow during the pulse allows a smooth transition to a majority argon or air plasma at high pressure, sustained by lower levels of radio frequency power.

\section{ACKNOWLEDGMENTS}

This research was supported by AFOSR Grant No. F49620-00-1-0181 and in part by NSF Grant No. ECS9905948. The authors thank Dr. K. Akhtar for careful reading of the manuscript and for discussions. They thank Dr. K. Schoenbach of Old Dominion University for loaning them the $105 \mathrm{GHz}$ interferometer.

${ }^{1}$ H. U. Eckert, F. L. Kelly, and H. N. Olsen, J. Appl. Phys. 3, 39 (1968).

${ }^{2}$ M. Larousi, Int. J. Infrared Millim. Waves 16, 2069 (1995).

${ }^{3}$ M. Larousi, Int. J. Infrared Millim. Waves 17, 2215 (1996).

${ }^{4}$ R. J. Vidmar, IEEE Trans. Plasma Sci. 18, 733 (1990).

${ }^{5}$ K. Kelly-Wintenberg, T. C. Montie, C. Brickman, J. R. Roth, A. K. Carr, K. Sorge, L. Wadsworth, and P. Tsai, J. Ind. Microbiol. Biotechnol. 20, 69 (1998).

${ }^{6}$ P. Tsai, L. Wadsworth, and J. R. Roth, Text. Res. J. 67, 359 (1997).

${ }^{7}$ A. Schuetze, J. Y. Young, S. E. Babayan, J. Park, G. S. Selwyn, and R. F. Hicks, IEEE Trans. Plasma Sci. 26, 1685 (1998).

${ }^{8}$ H. Kim and S. H. Hong, IEEE Trans. Plasma Sci. 23, 852 (1995)

${ }^{9}$ G. Babat, J. Inst. Electr. Eng., Part 1 94, 27 (1947).

${ }^{10}$ K. L. Kelly, J. E. Scharer, G. Ding, M. Bettenhausen, and S. P. Quo, J. Appl. Phys. 85, 63 (1999).

${ }^{11}$ G. Ding, J. E. Scharer, and K. L. Kelly, J. Appl. Phys. 84, 1236 (1998).

${ }^{12}$ G. Ding, J. E. Scharer, and K. L. Kelly, Phys. Plasmas 8, 334 (2001).

${ }^{13}$ S. Toby, P. A. Astheimer, and F. S. Toby, J. Photochem. Photobiol., A 67, 1 (1992).

${ }^{14}$ Y. Mouzouris and J. E. Scharer, IEEE Trans. Plasma Sci. 24, 152 (1996).

${ }^{15}$ Y. Mouzouris and J. E. Scharer, Phys. Plasmas 1, 875 (1998).

${ }^{16}$ Y. Mouzouris, Ph.D. dissertation, University of Wisconsin-Madison, 1998.

${ }^{17}$ W. Shen, J. E. Scharer, N. T. Lam, B. G. Porter, and K. L. Kelly, J. Appl. Phys. 78, 6974 (1995)

${ }^{18}$ V. Vahedi, R. A. Stewert, and M. A. Lieberman, J. Vac. Sci. Technol. A 11, 1275 (1993)

${ }^{19}$ R. A. Holroyd, J. M. Preses, C. L. Woody, and R. A. Johnson, Nucl. Instrum. Methods Phys. Res. A 261, 440 (1987).

${ }^{20}$ U. Kortshagen, N. D. Gibson, and J. E. Lawler, J. Phys. D 29, 1224 (1996)

${ }^{21}$ K. A. MacKinnon, Philos. Mag. 8, 605 (1929).

${ }^{22}$ K. Chandrakar, J. Phys. D 11, 1809 (1978).

${ }^{23}$ J. Amorim, H. S. Maciel, and J. P. Sudano, J. Vac. Sci. Technol. B 9, 362 (1991)

${ }^{24}$ M. A. Lieberman and A. J. Lichtenberg, Principles of Plasma Discharges and Materials Processing (Wiley, New York, 1994).

${ }^{25}$ O. A. Popov, High Density Plasma Sources (Noyes, Park Ridge, NJ, 1995). 
${ }^{26}$ Y. Nakato, M. Ozaki, A. Egawa, and H. Tsubomura, Chem. Phys. Lett. 9, 615 (1971).

${ }^{27}$ G. Ding, Ph.D. dissertation, University of Wisconsin-Madison, 1999.

${ }^{28}$ K. R. Stalder, R. J. Vidmar, and D. J. Eckstrom, J. Appl. Phys. 72, 5089 (1992).

${ }^{29}$ M. Wojcik and M. Tachiya, J. Chem. Phys. 110, 10016 (1999).

${ }^{30}$ M. J. Seaton, Mon. Not. R. Astron. Soc. 119, 81 (1959).

${ }^{31}$ B. D. McVey, Report PFC/RR-84-12, Plasma Fusion Center, MIT, 1984.
${ }^{32}$ B. D. McVey, Report PFC/RR-84-13, Plasma Fusion Center, MIT, 1984.

${ }^{33}$ K. L. Kelly, Ph.D. dissertation, University of Wisconsin-Madison, 2001.

${ }^{34}$ X. Guo, J. E. Scharer, J. Mouzouris, and L. Louis, Phys. Plasmas 6, 3400 (1999).

${ }^{35}$ Y. P. Raizer, M. N. Shneider, and N. A. Yatsenko, Radio-Frequency Capacitive Discharges (Chemical Rubber, Boca Raton, FL, 1995).

${ }^{36}$ J. J. Thompson, Philos. Mag. 23, 449 (1912). 